\title{
Free UH- $Q$ microtoroids, new tools for designing photonic devices
}

\author{
Mani Hossein-Zadeh, Kerry J. Vahala \\ T. J. Watson Laboratory of Applied Physics, California Institute of Technology, Pasadena, California, 91125, USA \\ vahala@caltech.edu,http://www.vahala.caltech.edu
}

\begin{abstract}
We describe techniques that enable fabrication of free UH- $Q$ silica microtoroids. Preliminary results show that free resonators with $Q$ s above 30 million can be fabricated and transferred to different platforms for integration with photonic devices.

(C)2007 Optical Society of America

OCIS codes: (220.4000) Optical fabrication; (230.5750) Resonators
\end{abstract}

\section{Introduction}

Ultrahigh- $Q$ (UH- $Q$ ) microtoroidal silica resonators represent a distinct class of optical microring resonators with $Q$ s in excess of 100 million [1]. The UH- $Q$ operation makes these devices very attractive for applications where low loss, large circulating power, small mode volume and narrow-linewidth are required. Employing UH- $Q$ microtoroids can dramatically improve the performance of multi-pole add-drop optical filters [2] and resonator based optical biosensors, enable the design of low threshold and narrow linewidth lasers and facilitate cavity QED experiments [3]. Unfortunately the special fabrication process of these microtoroids limits the range of possible integration choices for these resonators. Specifically, the diameter of the microdisk shrinks down as it reflows to its final toroidal shape and after fabrication, microtoroids are physically perched atop a silicon pillar. So those photonic devices that rely on coupling of these resonators to an integrated waveguide or their mutual coupling cannot be realized. Here we describe techniques that may enable fabrication of a new class of photonic devices based on free UH- $Q$ microresonators. Preliminary results show that by employing simple techniques we can detach the microtoroid from the silicon pillar without any damage to the microtoroid structure and maintain their quality factor above 10 million.

\section{Free ultra-high- $Q$ microtoroids}

After the conventional fabrication process [1], the silica microtoroid is perched atop a $20-40 \mu \mathrm{m}$ silicon pillar. To enable safe detachment of the silica microtoroid we reduce the diameter of the silicon pillar down to about 2-4 $\mu \mathrm{m}$ through a second $\mathrm{XeF}_{2}$ dry etching process (Fig. 1(a)). We have designed and fabricated special tools called 'microforks' for detachment and transfer of the microtoroids.
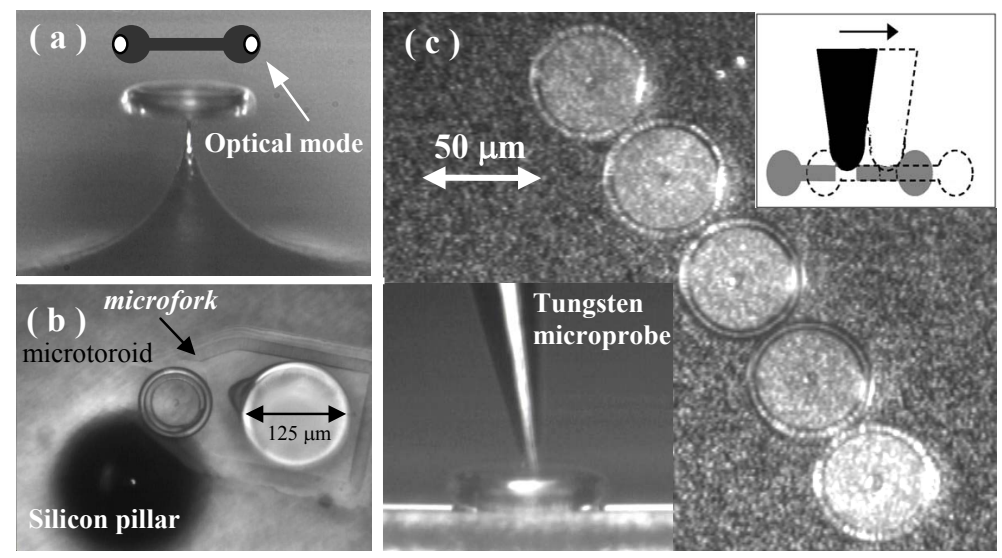

Fig. 1. (a) Photograph of a silica microtoroid with a narrow silicon pillar after second $\mathrm{XeF}_{2}$ etch. The cross-sectional diagram shows the location of the optical mode. (b) Top-view photograph of a free microtoroid detached from the silicon pillar using a silica microfork. The microfork is attached and controlled by a bare single mode fiber (white circle on the photograph). The microtoroid is sitting on the prongs of the microfork (c) Five, free microtoroids placed on the silicon substrate. These microtoroids are positioned using a Tungsten microprobe (See the top and bottom inset). 


\section{CFQ3.pdf}

Fig. 1(b) is the top view photograph of a microtoroid detached from the silicon pillar using the microfork. The prongs of the microfork are positioned under the microtoroid and the silicon-silica joint is broken by applying a vertical force. The detachment from the pillar doesn't damage the microring structure and only leaves a small hole (4-6 $\mu \mathrm{m}$ diameter) in the center of the 2-micron thick silica membrane (later this hole is used for accurate positioning of the microtoroid). Fig. 1(c) shows five, free microtoroids that are positioned side-by-side on the silicon substrate. These microtoroids are positioned using the Tungsten probe (shown in the bottom inset) that was controlled by a manual 3-D a nanopositoning system. The diagram in the top inset shows how the probe (that has a footprint of $3 \mu \mathrm{m}$ ) controls the microtoroid by insertion into the central hole.

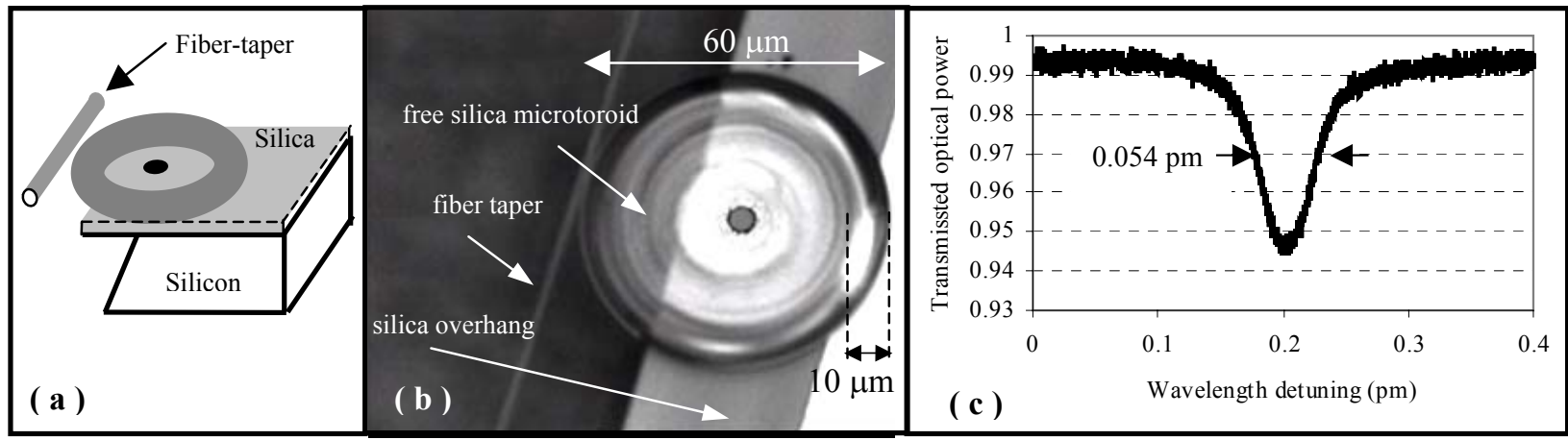

Fig. 2. (a) Schematic diagram of fiber-taper coupling to a free UH- $Q$ silica microtoroid optical resonator. The microtoroid is sitting on a 2micron thick silica membrane (on top of a silicon pedestal) to facilitate the side coupling to the fiber-taper. (b) Photograph of a free microtoroid on silica substrate coupled to a fiber-taper. The gray area in the photograph is the silica overhang and the white area is the silicon pedestal seen through the silica layer. (c) Optical transmission spectrum of the fiber-taper coupled to the free microtoroid near $1550 \mathrm{~nm}$. The measured quality factor $\left(Q_{\text {tot }}\right)$ is about $2.95 \times 10^{7}$ (given that at resonance the transmission is about $945 \%$ the corresponding $Q_{0}$ is $3.08 \times 10^{7}$ ).

Standard fiber-taper coupling techniques are used to measure the optical quality factor of the free microtoroid. We have designed a special platform that facilitates the fiber-taper coupling to a free microtoroid. This platform consists of a $2 \mu \mathrm{m}$ thick silica membrane supported by a silicon pedestal (about $30 \mu \mathrm{m}$ thick). Fig. 2(a) shows a schematic diagram of the coupling configuration. Figure 2(b) is the photograph of a free microtoroid on silica substrate coupled to a fiber-taper. Before detachment the measured quality factor $\left(Q_{\text {tot }}\right)$ of the microtoroid was about $5 \times 10^{7}$ corresponding to an unloaded quality factor $\left(Q_{0}\right)$ of $5.23 \times 10^{7}$. Fig. $2(\mathrm{c})$ shows the transmission spectrum of the fiber-taper coupled to the free microtoroid near $1550 \mathrm{~nm}$. After detachment the measured quality factor $\left(Q_{\mathrm{tot}}\right)$ is about $2.87 \times 10^{7}$ corresponding to an unloaded quality factor of $3.1 \times 10^{7}$. The coupling factor is set to small values to minimize the loading effect of the fiber-taper. Although the optical- $Q$ of the free microtoroid is degraded $(\sim 40 \%)$ the final $Q$ is still above 10 million. We believe the $Q$-degradation is mainly caused by contamination (since the whole process is performed in the lab environment and not in a clean room). Note that due to the sidewall curvature and thickness of the microtoroid cavity the optical mode does not interact with the substrate. This is an important feature that enables the integration of these resonators on almost any substrate. Currently, in all integrated microring resonators, the lateral optical confinement is provided by the index contrast rather than sidewall curvature consequently they must be fabricated on substrates with lower refractive index than the ring itself. This requirement sets a major limit on the variety of platforms and material systems that can be used in microring structures.

\section{Conclusion}

In conclusion we have demonstrated a practical technique for fabrication of free UH- $Q$ microtoroids. Free microtoroids can add the advantages of $\mathrm{UH}-Q$ operation to a variety of photonic devices. Beyond enabling the free microtoroid technology, our approach introduces the concept of fabrication and manipulation of free microresonators to photonic technology. We believe the extra degree of freedom offered by this approach can significantly increase the variety of possible configurations for photonic devices and improve their performance.

\section{References}

[1] Deniz D. K. Armani, T. J. Kippenberg, S. M. Spillane and K. J. Vahala, "Ultra-high-Q toroid microcavity on a chip," Nature 421, $925-929$ (2003).

[2] H. Rokhsari, and K. J. Vahala, "Ultralow loss, high-Q, four port resonant couplers for quantum optics and photonics," Phys. Rev. Lett. 92, 253905 (2004)

[3] T. Aoki, B. Dayan, E. Wilcut, W. P. Bowen, A. S. Parkins, T. J. Kippenberg, K. J. Vahala, and H. J. Kimble, "Observation of strong coupling between one atom and a monolithic microresonator," Nature 443, 671-674 (2006). 